\title{
Análisis Topográfico de la Superfície de Implantes de Titanio Comercialmente Puro a Través de Microscopía Electrónica de Barrido
}

\author{
Topographic Analysis of the Surface of Commercially \\ Pure Titanium Implants by Scanning Electronic Microscopy \\ Sandra de Cássia Santana Sardinha*; Antonio Gabriel Lanata-Flores*; Leandro Pozzer; \\ Lucas Cavalieri-Pereira*; Sergio Olate* \& José Ricardo Albergaria-Barbosa*
}

SARDINHA, S. C. S.; LANATA-FLORES, A. G.; POZZER, L.; CAVALIERI.PEREIRA, L.; OLATE, S. \& ALBERGARIABARBOSA, J. R. Análisis topográfico de la superficie de implantes de titanio comercialmente puro a través de microscopia electrónica de barrido. Int. J. Odontostomat., 7(1):125-131, 2013.

RESUMEN: Este trabajo tiene como objetivo analizar topográficamente la superficie de implantes de titanio comercialmente puros. Fueron seleccionados ocho implantes de las siguientes fabricantes: Conexão - Sistema de Próteses; Lifecore - Biomedical; AS Technology y S - Serson Internacional. Las muestras presentaban tratamiento de la superficies, a través de las técnicas de ataque acido (Conexão) y plasma spray de titanio (Lifecore-Biomedical; AS Technology y S Serson International), siendo divididas en cuatro grupos, conteniendo dos implantes del mismo lote de cada fabricante. Los análisis fueron realizados a través de microscopia electrónica de barredura (MEB). Los estudios a través de MEB revelaron la topografía rugosa de la superficie y la uniformidad de las áreas entre las roscas de los implantes. Se puede concluir que todas las muestras presentaron rugosidad en la superficie, con diferencias en la micromorfología entre todos los implantes.

PALABRAS CLAVE: implantes dentarios, titanio, microscopio electrónico de barrido.

\section{INTRODUCCIÓN}

Uno de los objetivos mas importantes de los implantes dentales es el restablecimiento de la función soportando fuerzas de diferentes magnitudes cuando se encuentran en función y en contacto con el hueso adyacente; una correcta orientación y magnitud junto a la fisiología ósea ideal optimiza los resultados (Wennerberg et al., 1995). Worthington et al. (1994), señalaron que un implante es considerado oseointegrado cuando exista una conexión directa entre el hueso y el titanio, la cual debe ser soportada en condiciones de carga. De esta forma, uno de los objetivos para el tratamiento de la superficie de los implantes es el aumentar el área de contacto con el tejido óseo, obteniendo una estabilidad ósea adecuada a través de la formación de rugosidad en la superficie (Taborelli et al., 1997).

El estudio criterioso de las técnicas de tratamiento de las superficies de los implantes de titanio debe considerar la posibilidad de contaminación de la superficies tanto en el empleo de las diversas formas de producción como también en el proceso de limpieza y esterilización (Henry, 1987; Swart et al., 1992; Li et al., 2001).

La técnica de microscopía electrónica de barrido (MEB) ha sido utilizada para caracterizar la topografía de superficie de diversos tipos de material y en diferentes condiciones de trabajo (Darvell et al., 1995) de forma que el objetivo de esta investigación es analizar la superficie de implantes con diferentes condiciones de fabricación con técnicas MEB.

\section{MATERIAL Y MÉTODO}

Se diseño un estudio descriptivo para el análisis de 8 implantes de titanio comercialmente puro con tratamientos de superficie (Tabla I) formando 4 grupos de trabajo para cada marca comercial con dos implantes de la misma marca en cada grupo:

\footnotetext{
* División de Cirugía Oral y Maxilofacial, Facultad de Odontología de Piracicaba Universidad Estadual de Campinas, Piracicaba, Brasil.

* Unidad de Cirugía Oral y Maxilofacial, Departamento de Odontología Integral Adultos, Facultad de Odontología, Universidad de La Frontera, Chile
} 
SARDINHA, S. C. S.; LANATA-FLORES, A. G.; POZZER, L.; CAVALIERI.PEREIRA, L.; OLATE, S. \& ALBERGARIA-BARBOSA, J. R. Análisis topográfico de la superficie de implantes de titanio comercialmente puro a través de microscopia electrónica de barrido. Int. J. Odontostomat., 7(1):125-131, 2013.

Tabla I. Especificaciones de producción de implantes según información del fabricante (Conexão - Sistemas de Próteses, Brasil. Lifecore Biomedical, Inc., USA. AS Technology , Brasil. S - Serson Internacional, Brasil).

\begin{tabular}{lllc}
\hline Grupo & Marca Comercial & Implante & Tratamiento de superficie \\
\hline 1 & Conexão & Master Porous ${ }^{\circledR}$ & Ataque Acido \\
2 & Lifecore Biomedical & Restore $®$ & Plasma Spray de Titanio (TPS) \\
3 & As Technology & Titanium Fix® & Plasma Spray de Titan io (TPS) \\
4 & S-Serson Implant & S-Serson $®$ & Plasma Spray de Titan io (TPS) \\
\hline
\end{tabular}

Los implantes fueron utilizados según la forma de venta con los envoltorios y elementos que tradicionalmente se encuentran en el mercado comercial; a través de un montador especifico para cada implante se procedió al retiro del mismo evitando contacto con cualquier tipo de superficie.

Se utilizó el microscopio electrónico de barrido JOEL-JSM, modelo 5600LV, donde fueron empleados valores diferentes de aceleración y distancia focal de acuerdo con el índice de aumento deseado de la imagen. Los datos referentes a la aceleración fueron dados en electro voltaje (Ev) y la distancia focal en milímetros (mm). Los implantes fueron posicionados sobre portamuestras sin que la superficie fuese manipulada por el
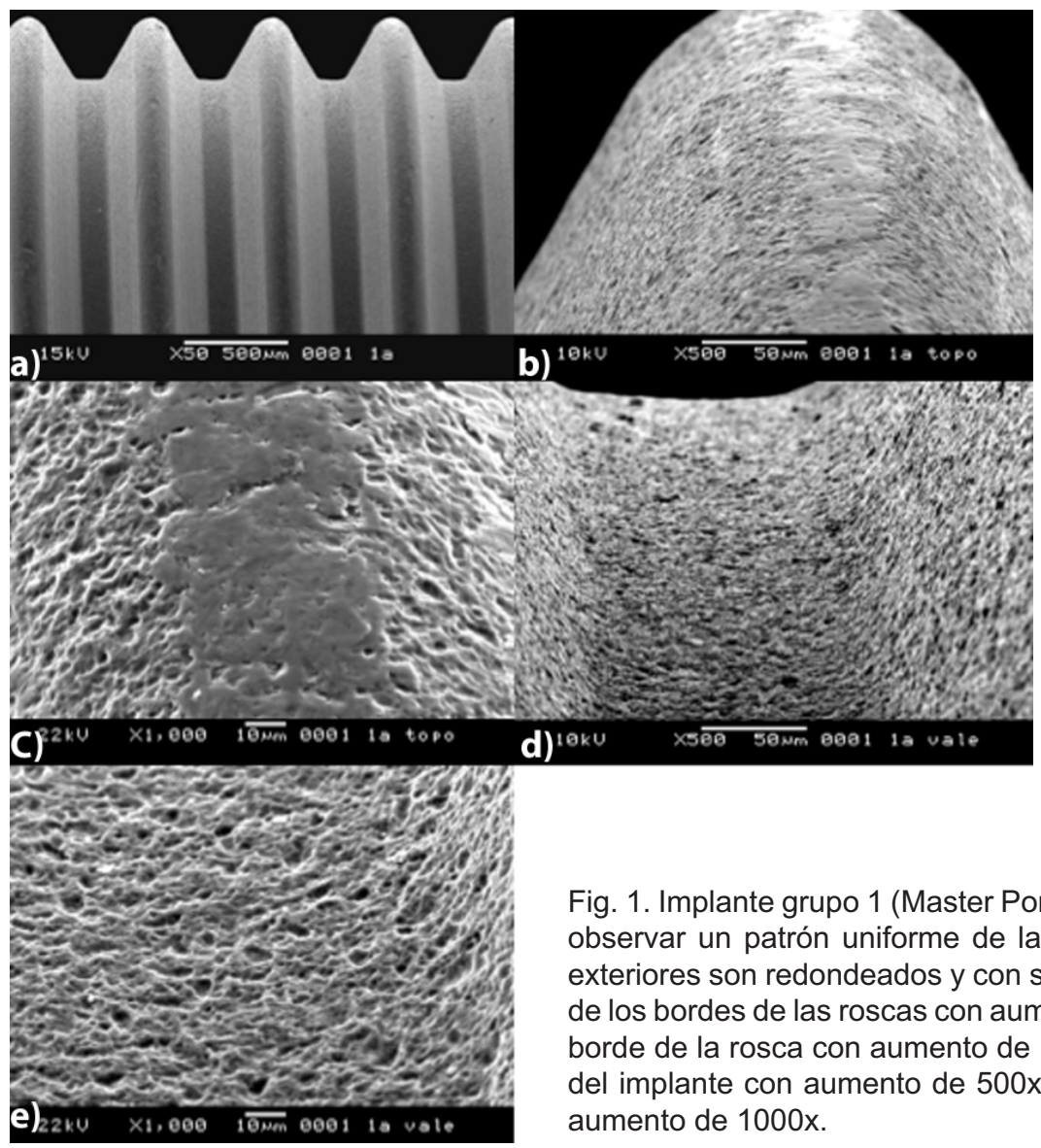
d) ${ }^{10 \mathrm{kU}}$

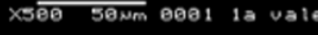
aumento de 1000x. operador. Fueron obtenidas diferentes imágenes (50x; 500x; 1000x) para identificar diferentes patrones del implante.

Las imágenes fueron captadas usando Kilovoltage de $10 \mathrm{Kv}, 15 \mathrm{Kv}$ o 22kv; el diámetro de sonda utilizada (spotsize) fue de 35 -38 y la distancia de trabajo fue de 32 a $35 \mathrm{~mm}$ conforme el aumento deseado de la imagen.

\section{RESULTADOS}

Al analizar los implantes de los cuatro grupos, se observa los diferentes aspectos en la topografía de las superficies, tanto en los valles como en el sector más exterior de las roscas, debido a los diferentes tratamientos realizados en los implantes por los fabricantes.

En los implantes del grupo 1 se puede observar al aumento de 50x (Fig. 1a) la uniformidad entre las roscas de los implantes. En una visión más aproximada, se usó aumentos de 500x (Fig. 1b) y 1000x (Fig. 1c) observando en el borde de las roscas, porosidades asociados al tratamiento de la superficie, en este caso por ataque acido; además se logra observar uniformidad en el aspecto poroso de las paredes laterales de la rosca. Usando los mismos aumentos en la región profunda de las roscas (Fig. 1d y

Fig. 1. Implante grupo 1 (Master Porous $®$ ). 1a) Aumento de 50x, donde se puede observar un patrón uniforme de las roscas, como también en los bordes mas exteriores son redondeados y con superficie regular. 1b) Micrografía de la región de los bordes de las roscas con aumento de 500x. 1c) Micrografía de la región del borde de la rosca con aumento de 1000x. 1d) Micrografía de la región del surco del implante con aumento de 500x. 1e) Micrografía de la región del surco con 
1e), se verifica un patrón regular y homogéneo de las porosidades, sin evidencia de áreas planas.

En el grupo 2, en el aumento de 50x (Fig. 2a), se observa un patrón uniforme de las roscas, con formas redondeadas e irregulares en el contorno, con sectores de mayor o menor profundidad. En análisis mas próximo del implante con imagen de 500x y 1000x (Fig. 2b-e), se logra identificar los grados de rugosidad en las superficies de los implantes, consecuente al tratamiento de plasma spray de titanio; se observaron rugosidades de $22-88 \mu \mathrm{m}$, con ausencia de espacios o grietas en la superficie. En este método, el titanio en polvo es aplicado usando un proceso especial de plasma spray, promoviendo una cobertura con alto grado de pureza.

Al análisis del grupo 3, en una vista panorámica de las roscas se observo a 50x (Fig. 3a), un padrón uniforme en los ángulos, presentando también bordes afilados y regularidad entre una y otra rosca. En imágenes obtenidas de los implantes del grupo 3, con aumento de 500x (Fig. 3b) y 1000x (Fig. 3c), se puede observar la rugosidad producida por el tratamiento empleado por el fabricante; también en imágenes aumentadas de los valles s observan (Fig. $3 \mathrm{~d}$ y $3 e)$ zonas lobuladas en la superficie del implante.

En un aumento de 50x, el análisis de los implantes del grupo 4 presentaba surcos uniformes en las superficies de la muestra (Fig. 4a). Imágenes más aproximadas con un aumento de 500x y de 1000x de las regiones de límites de roscas y valles del implante (Fig. $4 \mathrm{~b}-\mathrm{e})$, muestran rugosidad en la superficie con áreas planas y con pequeños relieves del tratamiento de superficie con el plasma Spray de titanio.
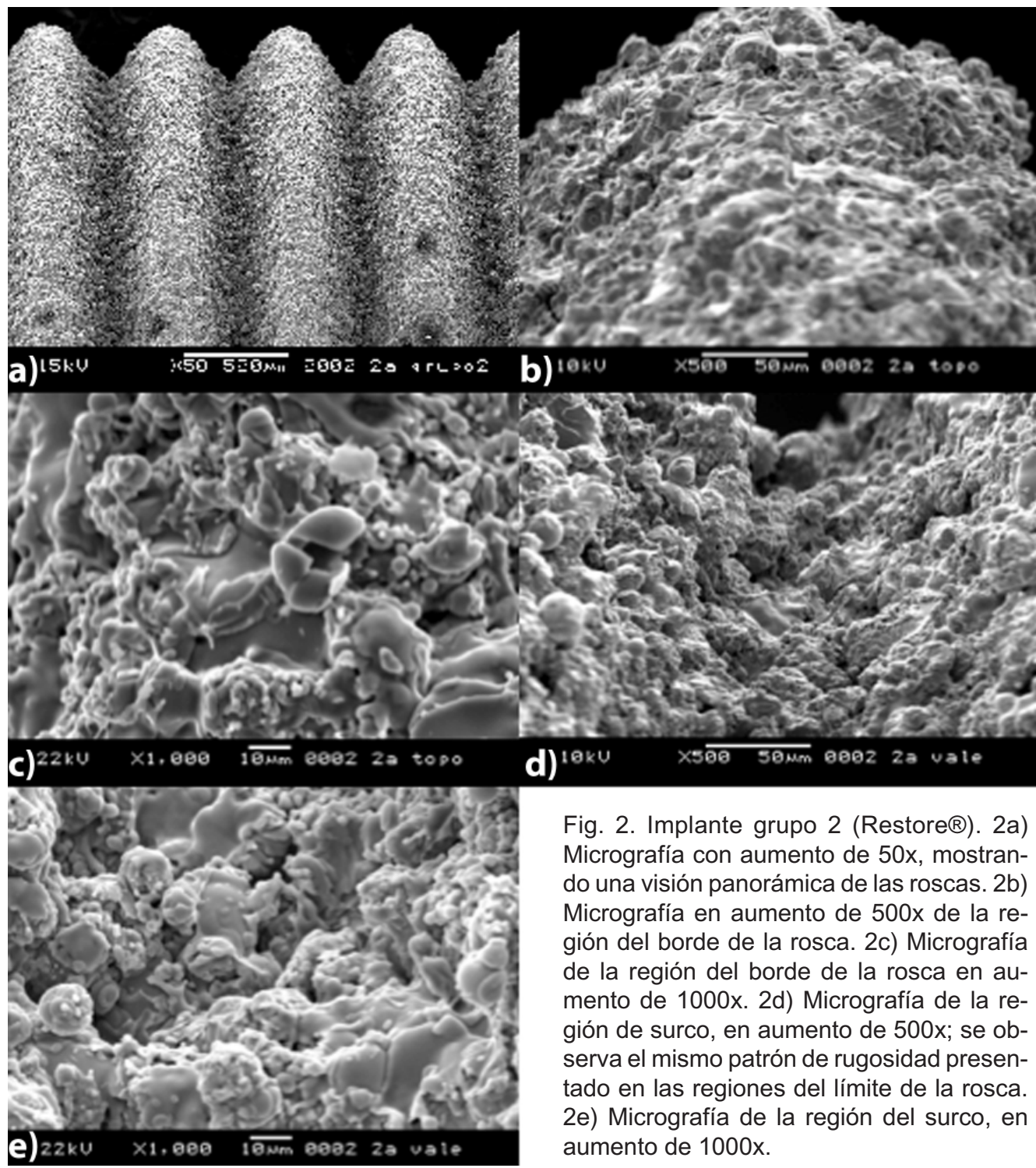

Fig. 2. Implante grupo 2 (Restore $®)$. 2a) Micrografía con aumento de 50x, mostrando una visión panorámica de las roscas. $2 b$ ) Micrografía en aumento de 500x de la región del borde de la rosca. 2c) Micrografía de la región del borde de la rosca en aumento de 1000x. 2d) Micrografía de la región de surco, en aumento de 500x; se observa el mismo patrón de rugosidad presentado en las regiones del límite de la rosca. 2e) Micrografía de la región del surco, en aumento de 1000x. 

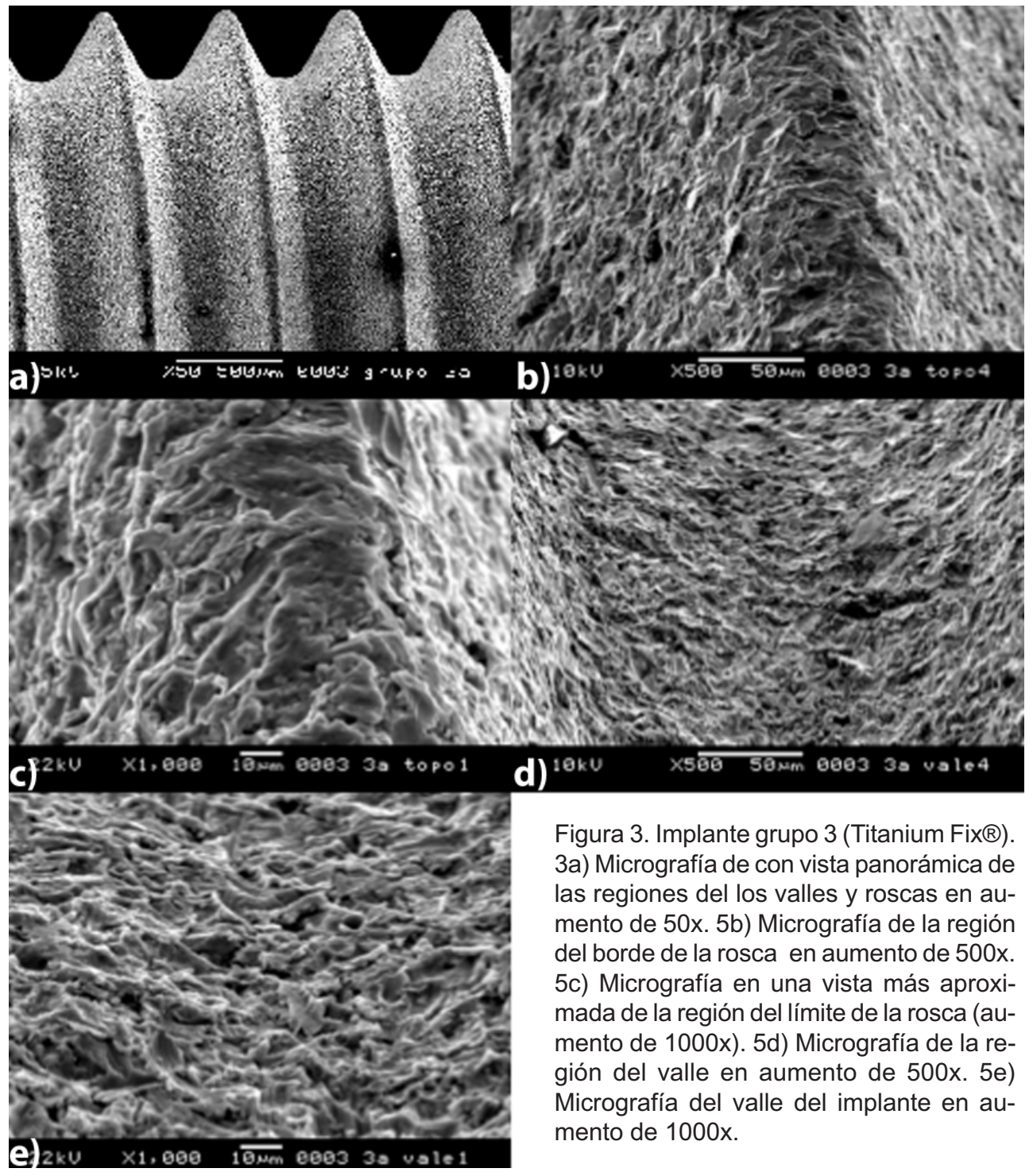

Figura 3. Implante grupo 3 (Titanium Fix®). 3a) Micrografía de con vista panorámica de las regiones del los valles y roscas en aumento de 50x. 5b) Micrografía de la región del borde de la rosca en aumento de 500x. 5c) Micrografía en una vista más aproximada de la región del límite de la rosca (aumento de 1000x). 5d) Micrografía de la región del valle en aumento de 500x. 5e) Micrografía del valle del implante en aumento de 1000x.

\section{DISCUSIÓN}

La MEB es una técnica que permite la visualización directa y rápida de la superficie de los implantes, permitiendo aumentos de hasta $200.000 x$, lo cual entrega una versatilidad muy elevada a estos sistemas. El sistema de análisis consiste en un mecanismo de emisión de electrones generado por un filamento de tungsteno, en un ambiente al vacío, que barre la superficie de la muestra generando las imágenes características. Este método también a sido utilizado por otros autores mostrando buenos resultados en la imagen de análisis (Darvell et al.; Ishikawa et al., 1997; Cooper et al., 1999; Leize et al., 2000; Orsini et al., 2000; Placko et al., 2000; Mustafa et al., 2001; Wieland et al., 2001).
El objetivo fundamental del tratamiento de superficie es el aumento de la superficie de contacto, con lo cual aumenta el área viable de oseointegración (Wennerberg et al.; Abron et al., 2001; Lim et al., 2001). Hansson \& Norton (1999) señalaron la necesidad de producir rugosidades que permitan la formación de tejido en la superficie de los implantes, donde las medidas entre 1 y $5 \mathrm{~mm}$ de diámetro fueron consideradas ideales. Aunque no fue el objetivo de esta investigación el análisis métrico de las rugosidades de superficie, si fue posible identificar en el grupo 2 grados de rugosidad de la superficie de entre 22 y $28 \mathrm{~mm}$. Por otra parte, estudios previos también han mostrado ta- 


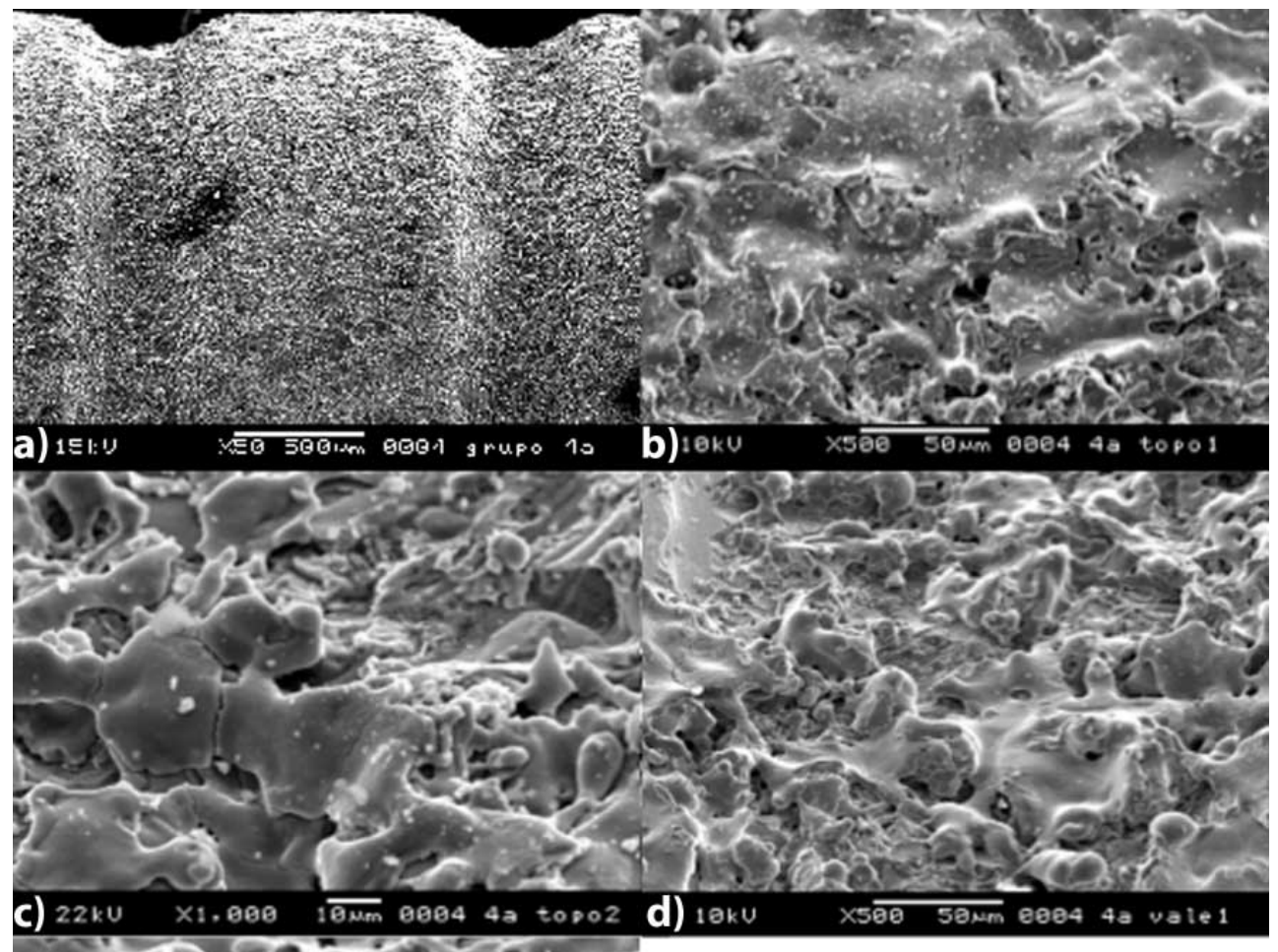

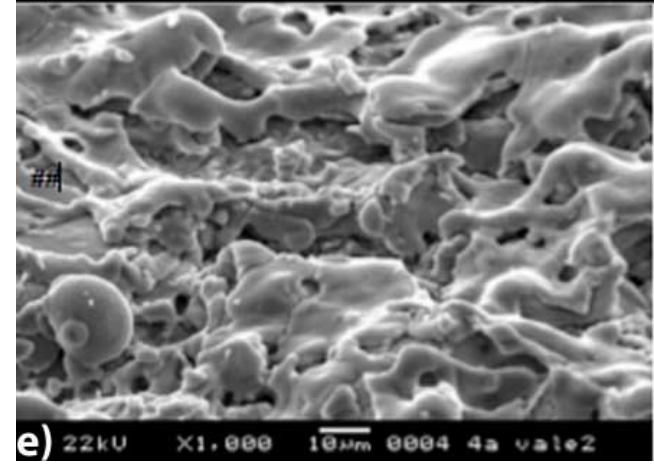

maños de entre $10 \mathrm{~mm}$ y $20 \mathrm{~mm}$ en implantes dentales de titanio maquinados sin tratamiento de superficie (Olate et al., 2010). La rugosidad del implante es importante para determinar el éxito clínico (Glauser et al., 2005), demostrando también que en implantes de rugosidades menores el torque de remoción de implantes es mayor (Gotfredsen et al., 2000); a pesar de que esta micromorfología es fundamental en el éxito clínico, otros elementos como la configuración de paredes, roscas y la macromorfología del implante son fundamentales en el éxito de la oseointegración (Jaimes et al., 2009; Olate et al., 2011).

El mecanismo de tratamiento a través de ataque ácido fue analizado por Li et al., quienes observaron modificaciones de la superficie, presentando microporos
Figura 4. Implante grupo 4 (S - Serson®). 4a) Micrografía con vista en aumento de 50x de la región de dos de los valles del implante. 4b) Micrografía en la región del límite de una rosca en aumento de 500x. 4c) Micrografía en vista más aproximada da región de rosca (aumento de 1000x). 4d) Micrografía de un implante en una región del valle en aumento de 500x 4e) Micrografía en región del valle, en un aumento de 1000x. de $2 \mathrm{~mm}$ de diámetro en la base del implante sin alterar la biocompatibilidad del mismo. La rugosidad producida por el ataque acido se mostraba regular, observándose también la eliminación de contaminantes de la superficie. Tres sistemas de implantes de nuestra muestra presentaron tratamiento con spray de plasma de titanio identificándose claras diferencias en términos de micromorfología; especificaciones técnicas de cada empresa son complejas de analizar debido a que no existe total información de los fabricantes en cuanto a los detalles de cada proceso de fabricación; se pueden identificar claras diferencias entre los implantes a pesar de realizar el mismo sistema de tratamiento. Cambios asociados de la duración del bombardeo de plasma, distancia del bombardeo $\mathrm{u}$ otras modificaciones pueden ser responsables de las diferencias entre los implantes. 
SARDINHA, S. C. S.; LANATA-FLORES, A. G.; POZZER, L.; CAVALIERI.PEREIRA, L.; OLATE, S. \& ALBERGARIA-BARBOSA, J. R. Análisis topográfico de la superficie de implantes de titanio comercialmente puro a través de microscopia electrónica de barrido. Int. J. Odontostomat., 7(1):125-131, 2013.

Finalmente, en base a la metodología empleada, se puede señalar que todos los implantes mostraron rugosidades de superficie, con características di- ferentes de tamaño y de forma de las rugosidades aunque el mecanismo de tratamiento de superficie fuese inicialmente el mismo.

SARDINHA, S. C. S.; LANATA-FLORES, A. G.; POZZER, L.; CAVALIERI.PEREIRA, L.; OLATE, S. \& ALBERGARIABARBOSA, J. R. Topographic analysis of the surface of commercially pure titanium implants by scanning electronic microscopy. Int. J. Odontostomat., 7(1):125-131, 2013.

ABSTRACT: The aim of this research is to analyze the pure titanium dental implant surface. An 8 implant device of Conexão - Sistema de Próteses; Lifecore - Biomedical; AS Technology y S - Serson Internacional was selected. The simple presented surface treatment with acid attack or titanium plasma spray, being analyzed in 4 groups with two implants in each. Those analyzed were realized with a scanning electron microscope showing the topography and structure between different part of the implant. It was concluded that all implant systems show surface roughness with differences in the micromorphology among all implant groups.

KEY WORDS: dental implant, titanium, scanning electronic microscopy.

\section{REFERENCIAS BIBLIOGRÁFICAS}

Abron, A.; Hopfensperger, M.; Thompson, J. \& Cooper, L. F. Evaluation of a predictive model for implant surface topography effects on early osseointegration in the rat tibia model. J. Prosthet. Dent., 85(1):40-6, 2001.

Cooper, L. F.; Masuda, T.; Whotson, S. W.; Yliheikkilä, P. \& Felton, D. A. Formation o mineralizing osteoblast cultures on machined, titanium oxide grit-blasted, and plasma-sprayed titanium surfaces. Int. J. Oral Maxillofac. Implants, 14(1):37-47, 1999.

Darvell, B. W.; Samman, N.; Luk, W. K.; Clark, R. K. \& Tideman, $\mathrm{H}$. Contamination of titanium castings by aluminium oxide blasting. J. Dent., 23(5):319-22, 1995.

Glauser, R.; Ruhstaller, P.; Windisch, S.; Zembic, A.; Lundgren, A.; Gottlow, J.; et al. Immediate occlusal loading of Brånemark System TiUnite implants placed predominantly in soft bone: 4-year results of a prospective clinical study. Clin. Implant. Dent. Relat. Res., 7 Suppl 1:S52-9, 2005.

Gotfredsen, K.; Berglundh, T. \& Lindhe, J. Anchorage of titanium implants with different surface characteristics: an experimental study in rabbits. Clin. Implant Dent. Relat. Res., 2(3):120-8, 2000.

Hansson, S. \& Norton, M. The relation between surface roughness and interfacial shear strength for boneanchored implants. A mathematical model. J. Biomech., 32(8):829-39, 1999.

Henry, P. J. Comparative surface analysis of two osseointegrated implant systems. Int. J. Oral Maxillofac. Implants, 2(1):23-7, 1987.
Ishikawa, K.; Miyamoto, Y.; Nagayama, M. \& Asaoka, K. Blast coating method: new method of coating titanium surface with hydroxyapatite at room temperature. J. Biomed. Mater. Res., 38(2):129-34, 1997.

Jaimes, M.; Duque de Miranda Chaves Netto, H.; Olate, S.; Mazzonetto, R. \& Albergaria Barbosa, J. R. Análisis fotoelástico descriptivo de dos diferentes diseños de implantes endoóseos sometidos a carga de compresión vertical. Av. Periodon. Implantol., 21(3):135-40, 2009

Leize, E. M.; Hemmerlé, J. \& Leize, M. Characterization, at the bone cristal level, of the titanium-cating/bone interfacial zone. Clin. Oral Implants Res., 11(4):279-88, 2000.

Li, D.; Liu, B.; Han, Y. \& Xu, K. Effects of a modified sandblasting surface treatment on topographic and chemical properties of titanium surface. Implant Dent., 10(1):59-64, 2001

Lim, Y. J.; Oshida, Y.; Andres, C. J. \& Barco, M. T. Surface characterizations of variously treated titanium materials. Int. J. Oral Maxillofac. Implants, 16(3):333-42, 2001.

Mustafa, K.; Wennerberg, A.; Wroblewski, J.; Hultenby, K.; Lopez, B. S. \& Arvidson, K. Determining optimal surface roughness of $\mathrm{TiO}_{2}$ blasted titanium implant material for attachment, proliferation of cells derived from human mandibular alveolar bone. Clin. Oral Implants Res., 12(5):515-25, 2001.

Olate, S.; Duque de Miranda Chaves Netto, H. \& de Albergaria-Barbosa, J. R. Análisis microestructural de cinco sistemas de implantes de titanio comercialmente puro. Av. Periodon. Implantol., 22(1):37-43, 2010. 
SARDINHA, S. C. S.; LANATA-FLORES, A. G.; POZZER, L.; CAVALIERI.PEREIRA, L.; OLATE, S. \& ALBERGARIA-BARBOSA, J. R. Análisis topográfico de la superficie de implantes de titanio comercialmente puro a través de microscopia electrónica de barrido. Int. J. Odontostomat., 7(1):125-131, 2013.

Olate, S.; Chaves Netto, H. D.; Kluppel, L. E.; Mazzonetto, R. \& de Albergaria-Barbosa, J. R. Mineralized tissue formation associated with 2 different dental implant designs: histomorphometric analyses performed in dogs. J. Oral Implantol., 37(3):319-24, 2011.

Orsini, G.; Assenza, B.; Scarano, A.; Piattelli, M. \& Piattelli, A. Surface analysis of machined versus sandblasted and acid etched titanium implants. Int. J. Oral Maxillofac. Implants, 15(6):779-84, 2000.

Placko, H. E.; Mishra, S.; Weimer, J. J. \& Lucas, L. C. Surface characterization of titanium-based implant materials. Int. J. Oral Maxillofac. Implants, 15(3):355-63, 2000.

Swart, K. M.; Keller, J. C.; Wightman, J. P.; Draughn, R. A.; Stanford, C. M. \& Michaels, C. M. Short-term plasmacleaning treatments enhance in vitro osteoblast attachment to titanium. J. Oral Implantol., 18(2):130-7, 1992.

Taborelli, M.; Jobin, M.; Francois, P.; Vaudaux, P.; Tonetti, M.; Szmukler-Moncler, S.; et al. Influence of sface treatments developed for oral implants on the physical and biological properties of titanium.1.surface characterization. Clin. Oral Implants Res., 8(3):208-16, 1997.

Wennerberg, A.; Albrektsson, T.; Andersson, B. \& Krol, J. J. A histomorphometric and removal torque study of screwshaped titanium implants with three different surface topographies. Clin. Oral Implants Res., 6(1):24-30, 1995.

Wieland, M.; Textor, M.; Spencer, N. D. \& Brunette, D. M. Wavelength-dependent roughness: a quantitative approach to characterizing the topography of rough titanium surfaces. Int. J. Oral Maxillofac. Implants, 16(2):163-81, 2001.

Worthington, P.; Lang, B. R. \& Lavelle, C. E. Osseointegration in dentistry. Chicago, Quintessence, 1994. p.19.

\author{
Dirección para Correspondencia: \\ Prof. Dr. Sergio Olate \\ Unidad de Cirugía Oral y Maxilofacial \\ Universidad de La Frontera \\ Claro Solar No 115, Temuco \\ CHILE
}

Telefono: 56-45-2325000

Email: sergio.olate@ufrontera.cl

Recibido : 17-09-2012

Aceptado: 01-12-2012 